\title{
FAILURE BEHAVIOR OF RC STRUCTURES UNDER IMPACT LOADS BASED ON RIGID-BODY-SPRING MODELS
}

\author{
YOUNG KWANG HWANG* , JOHN E. BOLANDER ${ }^{\dagger}$ AND YUN MOOK LIM $^{\dagger \dagger}$ \\ ${ }^{*}$ Yonsei University \\ Seoul, Republic of Korea \\ e-mail: youngkwang@yonsei.ac.kr \\ ${ }^{\dagger}$ University of California, Davis \\ CA, USA \\ e-mail: jebolander@ucdavis.edu \\ 广YYonsei University \\ Seoul, Republic of Korea \\ e-mail: yunmook@yonsei.ac.kr
}

Key words: Rate dependency, Reinforced concrete, Dynamic fracture, Rigid-Body-Spring Network

\begin{abstract}
In this study, the dynamic behavior of reinforced concrete (RC) beams under impact loads is simulated considering the rate dependency of concrete mechanical properties. The mechanical properties (e.g. strength, elastic modulus, and fracture energy) of concrete increase under high rates of loading. This increase is often depicted in terms of a dynamic increase factor (DIF) curve. Generally, the DIF value rapidly increases in the regime of high strain rates, greater than $1 \mathrm{~s}^{-1}$. The rate dependency of concrete has to be considered in the simulation of concrete under impact load. Herein, a three-dimensional dynamic model, comprised of rigid-body-spring elements fitted with rheological units, is validated through comparison with existing experimental results. The validated model is used to simulate the failure of RC beams, which changes under high loading rates. It is shown that the severity of diagonal shear failure increases with loading rate. With further development, the model can be used to understand the behavior of RC structures subjected to various forms of impact loading.
\end{abstract}

\section{INTRODUCTION}

Concrete testing has been conducted under various loading rates at the material and structural levels [1-6]. As shown in Fig. 1, concrete strength varies according to the strain rate. The DIF curves exhibit two regimes. In the first regime, the DIF value gradually increases with strain rate. The second regime, beginning at about $1 \mathrm{~s}^{-1}$, exhibits a rapid increase in DIF with strain rate. This phenomena can be hypothetically explained by the Stefan effect at lower rates, related with the viscosity of free water within concrete microstructure, and inertial effects at higher loading rates [1].

High rate dependency occurs in the regime of high strain rate $\left(\dot{\varepsilon}>1 \mathrm{~s}^{-1}\right)$, which can be classified as the region of impact load (Fig. 2). This study focusses on impact loading. The high rate dependency of concrete is simulated by a visco-elasto-plastic damage (VEPD) unit composed of spring, dashpot, and Coulomb friction components (Fig. 3) [8-10]. The VEPD units are introduced within a rigid-body-spring network (RBSN), which is a form of lattice model. 


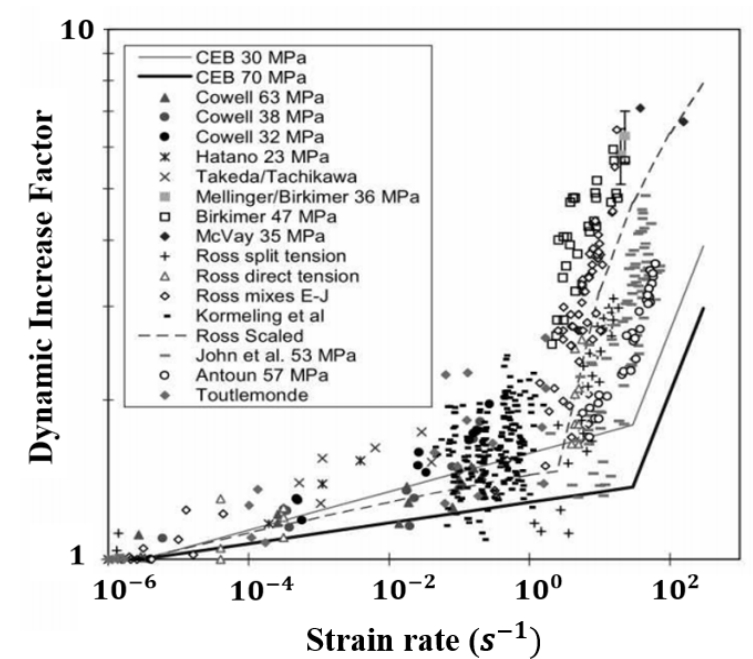

Figure 1: Experimental DIF curves for tensile strength [2]

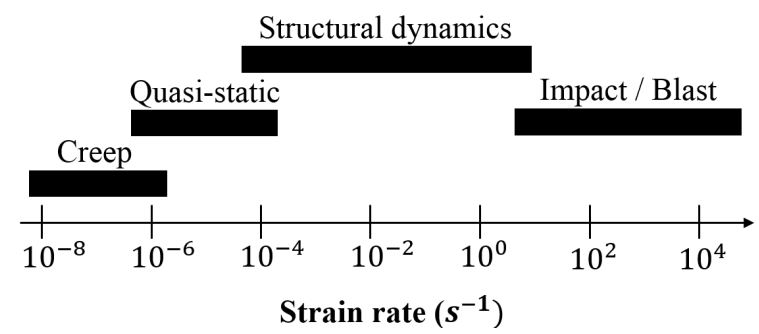

Figure 2: Regimes of mechanical responses for a wide range of strain rates [7]

RBSN models have been used to simulate the tensile fracture behavior of concrete both in static and dynamic analyses [11-12]. More general dynamic loading patterns can be accommodated by Lattice-Discrete Particle Models [13], which also offer explicit representations of material heterogeneity. For dynamic analysis presented herein, Verlet-like explicit time integration schemes are adopted [12]. The previously developed 2D model [7, 12] is extended to three dimensions, which enables consideration of 3D geometries, arrangement of reinforcement, and general loading patterns.

Capabilities of the 3D model are demonstrated by simulations of RC beams under impact loading. Model results are compared with existing experimental results, after which the model is used to examine failure behavior under high loading rate conditions.

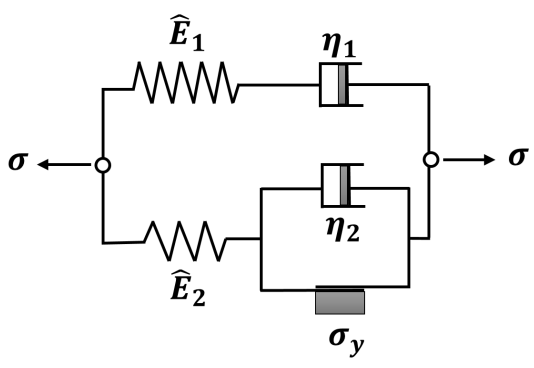

Figure 3: Configuration of VEPD unit [8]

\section{MODELING APPROACH}

The dynamic RBSN model is based on a Delaunay/Voronoi discretization of the structural domain. The discretization process, shown in Fig. 4, involves: 1) the random placement of a set of points within the domain; and 2) the Delaunay/Voronoi dual tessellation of the point set. Special considerations are used to define the boundaries. The rigid-body-spring elements connect neighboring nodes via a zerosize spring set (positioned at the area centroid of the Voronoi facet shared by the nodal pair) and rigid-arm constraints that link the spring set and the nodal degrees of freedom (Fig. 5) [11, 14]. Herein, each spring within the conventional RBSN is replaced with the VEPD unit shown in Fig. 3.

The steel reinforcing bars are discretized as rigid-body-spring elements. This requires the placement of nodes, representing the steel, in coordination with the nodes representing the concrete.

The reinforcing steel is assumed to be elastic-perfectly plastic. The steel is bonded to the concrete through an elastic interface; bond slip is not considered at this stage of program development. Concrete cracking is represented

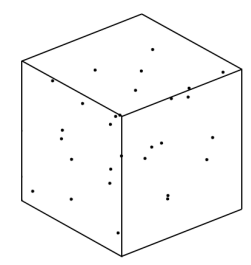

(a)

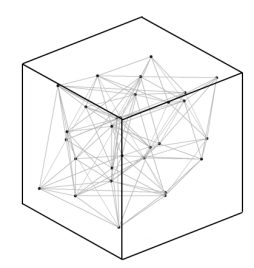

(b)

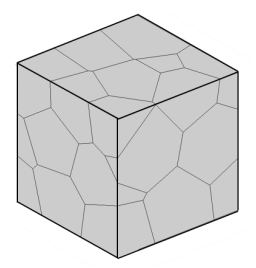

(c)
Figure 4: Domain discretization: (a) randomly generated points (b) Delaunay tessellation (c) Voronoi tessellation [15] 


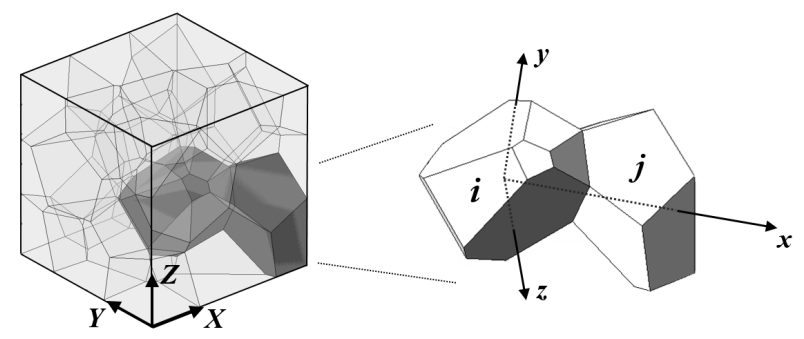

(a) two-cell assembly [10]

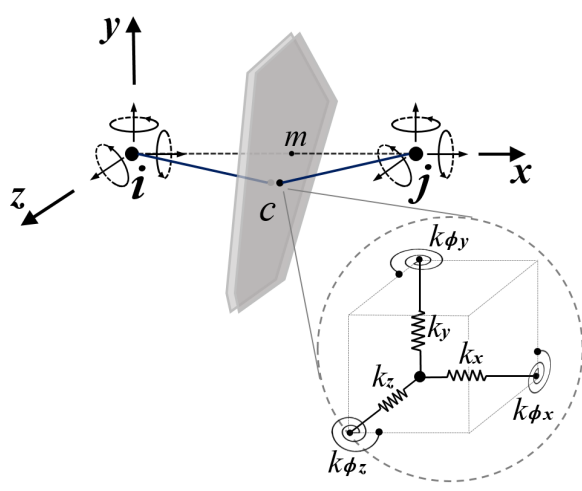

(b) RBSN element [16]

Figure 5: RBSN element configuration

by isotropic, bilinear softening of the spring sets within the critical rigid-body-spring elements. Criticality is based on the stress state of each spring set relative to a Mohr-Coulomb failure surface [11-12].

\section{VALIDATION EXERCISE}

As a first step toward model validation, a RC beam test of Kulkarni and Shah [5] is simulated. The four-point bend test configuration is shown in Fig. 6. Discretization of the test specimen involves about 3500 nodes and 24,000 elements (Fig. 7). In accordance with the test program, load on the model is applied under displacement control at a rate of $380 \mathrm{~mm} / \mathrm{s}$.

Fig. 8(a) depicts the experimental result. Failure occurred in the compression zone between the two loading points; distributed cracking appeared in the tension zone over much of the beam length. Fig. 8(b)-(c) present the simulation results, which agree well with previous two-dimensional simulation results [12]. The figures show damage zones within the simulated beam for two stages of the loading, according to imposed displacement $\Delta$.

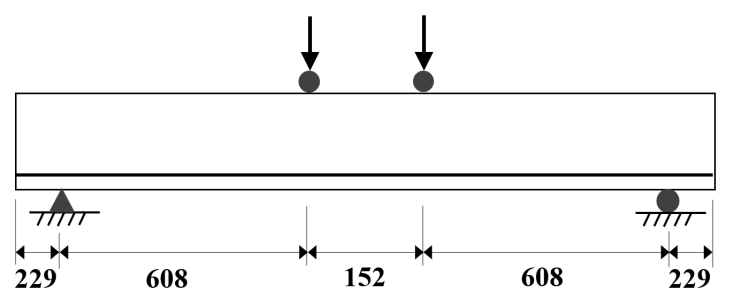

(a) load and boundary conditions

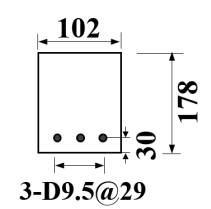

(b) cross-section

Figure 6: Experimental set up for four-point bending test [5]

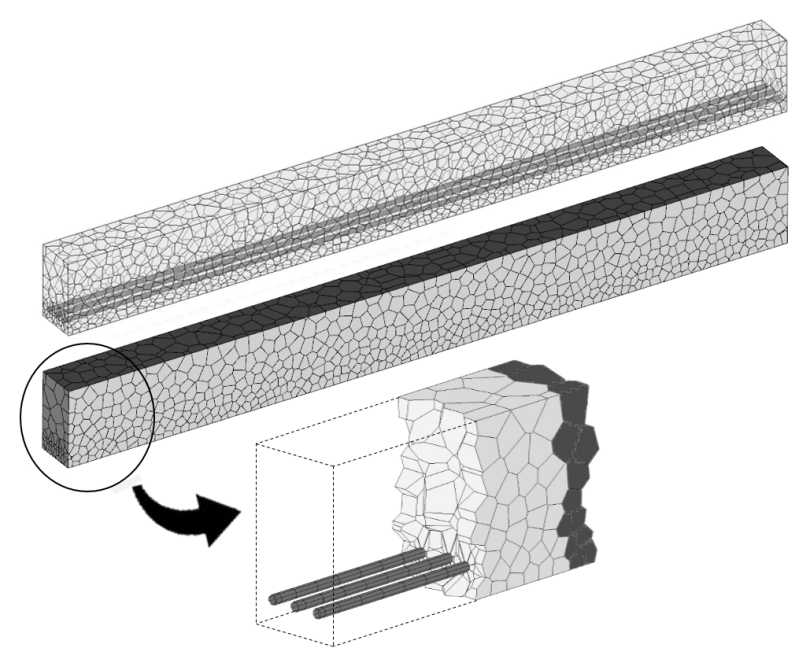

Figure 7: Internal and external views of the $\mathrm{RC}$ beam model

In the simulated test, cracks initiated from the central, bottom face of the beam (Fig. 8(b)). The smaller crack spacing, relative to that seen in the experiment, is likely due to the lack of bond slip in the simulation.

With continued loading, diagonal cracks form in the simulated beam (Fig. 8(c)). In the experiment, such shear failure was precluded by compressive failure of the concrete between the loading points. At the current stage of model development, the concrete is assumed to be linear elastic in compression. A more realistic representation of the concrete in compression would likely improve the correspondence between the simulated and actual test results. 


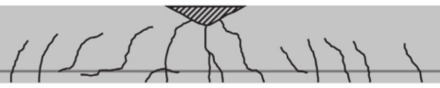

(a) Experimental result [5]

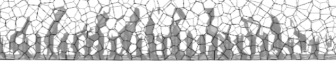

(b) $\Delta=3.8 \mathrm{~mm}$

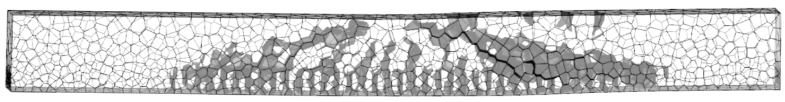

(c) $\Delta=8.4 \mathrm{~mm}$

Figure 8: Experimental and simulated crack patterns of $\mathrm{RC}$ beam

Results obtained with the previously developed 2D model [12] are reproduced in Fig. 9. Comparing the $2 \mathrm{D}$ and $3 \mathrm{D}$ simulation results, for the loading velocity of $380 \mathrm{~mm} / \mathrm{s}$, one sees that the overall damage patterns are similar. However, cracks within the 2D model are discrete in appearance; the 3D model exhibits both discrete-like and diffuse cracking. What appears to be a broadening of the damage zone in $3 \mathrm{D}$ is due to the random geometry of the RBSN in the thickness direction and a small degree of stress locking. The experimental results are presented in terms of surface cracking. If the experimental crack patterns could be seen in three dimensions, the elevation views of cracking would appear less discretelike.

\section{EFFECTS OF LOADING RATE}

Herein, a parametric study of the RC beam with three different loading rates $(380,1900$, and $3800 \mathrm{~mm} / \mathrm{s}$ ) is conducted. The beam has the same geometry and boundary conditions as depicted in Fig. 6. Fig. 10 shows the damage configurations of the beam under the three different loading rates; all results are for a central deflection of $\Delta=15.2 \mathrm{~mm}$. The results show a trend toward shear failure as loading rate increases, which was also described in [5].

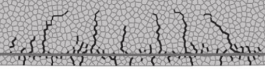

(a) $\Delta=3.8 \mathrm{~mm}$

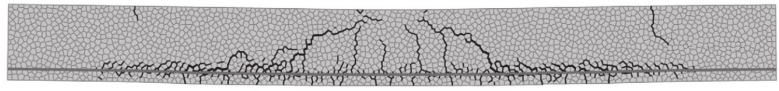

(b) $\Delta=8.4 \mathrm{~mm}$

Figure 9: Crack patterns in 2D simulation results [12]

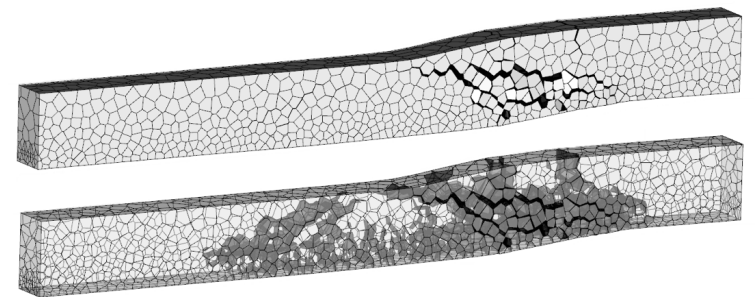

(a) $380 \mathrm{~mm} / \mathrm{s}$

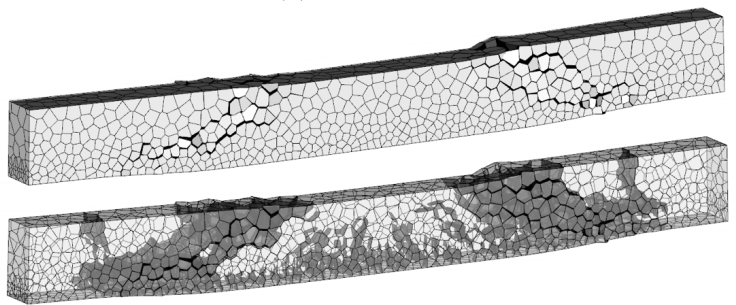

(b) $1900 \mathrm{~mm} / \mathrm{s}$

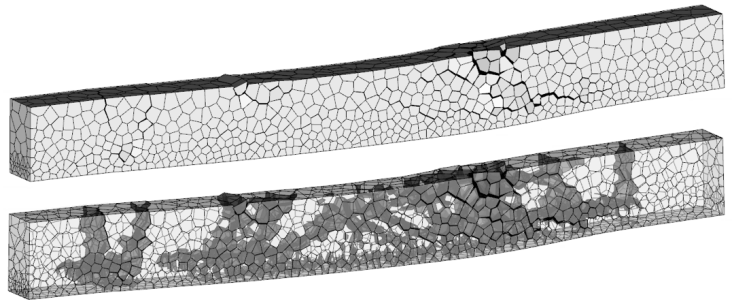

(c) $3800 \mathrm{~mm} / \mathrm{s}$

Figure 10: Simulated failure configurations for different loading rates

The parametric study indicates that, as expected, the failure mechanisms under impact-type loading are different from those under static loading.

Ongoing work involves model extensions (e.g., to accommodate concrete nonlinearity in compression and bond slip at the concrete-steel interface) and more rigorous validation exercises. A more general, validated model will enable study of the effects of loading rate, reinforcing steel arrangement, interface properties and projectile geometry.

\section{CONCLUSIONS}

In this study, the failure behavior of RC beams under impact loads is simulated using a newly developed RBSN model. The rate dependent features of concrete are represented by visco-elasto-plastic damage (VEPD) units, which take the place of the springs in the conventional RBSN.

To validate the newly developed model, simulation results of RC beams under impact loading are compared with experimental results 
and those of previous 2D models. The simulated and experimentally observed failure configurations are similar. However, the observed flexural cracks are more widely spaced along the length of the beam, relative to the simulation results, which is likely due to the assumption of no bond slip in the model. Furthermore, the assumption of concrete linearity in compression contributed to shear failure of the beam models. Concrete crushing in the compression zone of the test specimens likely precluded such shear failure.

After these validation efforts, additional numerical tests were conducted to study the failure trends of RC beams under high loading rates. As the loading rate increased, shear cracking becomes more severe and failure becomes more brittle.

In future study, the developed model will be extended to consider additional nonlinear behaviors, including those related to the concrete-steel interface and concrete behavior in compression. It is anticipated that the failure modes of RC beams can be accurately simulated, which will support the design of impact resistant structural concrete.

\section{ACKNOWLEDGMENTS}

This work was supported by the National Research Foundation of Korea (NRF) grant funded by the Korea goverment (MSIP) (No. 2014R1A2A2A01004421).

\section{REFERENCES}

[1] Rossi, P., Van Mier, J.G.M., Toutlemond F., Maou, F.L., and Boulay, C. 1994. Effect of loading rate on the strength of concrete subjected to uniaxial tension. Material and Structures 27:260-264.

[2] Malvar, L.J. and Ross, C.A. 1998. Review of strain rate effects for concrete in tension. ACI Materials Journal 95(6):735-739.

[3] Cadoni, E., Solomos, G., and Albertini, C. 2013. Concrete behavior in direct tension tests at high strain rates. Magazine of Concrete Research 65(11):660-672.

[4] Shah, S.P., Wang, M.L., and Chung, L. 1987. Model concrete beam-column joints subjected to cyclic loading at two rates.
Material and Structures 20:85-95.

[5] Kulkarni, S.M. and Shah, S.P. 1998. Response of reinforced concrete beams at high strain rates. ACI Structural Journal 95(6):705-715

[6] Adhikary, S.D., Li, B., and Fujikake, K. 2012. Dynamic behavior of reinforced concrete beams under varying rates of concentrated loading. International Journal of Impact Engineering 47:24-38.

[7] Kim, K. 2011. Development of irregular lattice models for simulating rate dependent failure in concrete materials and structures. PhD Thesis. Yonsei University, Seoul, Republic of Korea.

[8] Hwang, Y.K., Kim, K., Bolander, J.E., and Lim, Y.M. 2015. Evaluation of rheological models within lattice-based simulations of concrete under dynamic loading. In: Kodur, V.K.R. and Banthia, N. (Eds) Proceedings of $5^{\text {th }}$ International Workshop on Performance, Protection \& Strengthening of Structures under Extreme Loading; pp. 36-43.

[9] Hwang, Y.K. and Lim, Y.M. 2015. Rheological models for simulations of concrete under high-speed load. Journal of the Korean Society of Civil Engineers 35(4):769-777. (Korean)

[10]Hwang, Y.K., Bolander, J.E., and Lim, Y.M. 2016. Concrete failure simulation under high loading rates using threedimensional irregular lattice model. Mechanics of Materials (submitted)

[11]Bolander, J.E., Saito, S. 1998. Fracture analyses using spring networks with random geometry. Engineering Fracture Mechanics 61:569-591

[12] Kim, K., Bolander, J.E., and Lim, Y.M. 2013. Failure simulation of RC structures under highly dynamic conditions using random lattice models. Computers and Structures 125:127-136.

[13] Smith, J., Cusatis, G., Pelessone, D., Landis, E., O'Daniel, J. and Baylot, J. 2014. Discrete modeling of ultra-highperformance concrete with application to projectile penetration. International Journal of Impact Engineering 65:13-32. 
[14] Kawai, T. 1978. New discrete models and their application to seismic response analysis of structures. Nuclear Engineering and Design 48:207-229.

[15]Yip, M., Mohle, J., Bolander, J.E. 2005. Automated modeling of three-dimensional structural components using irregular lattices. Computer-Aided Civil and Infrastructure Engineering 20:393-407.

[16] Yip, M. 2005. Irregular lattice simulation of cement composite materials and structures. $\mathrm{PhD}$ Thesis. University of California, Davis, CA, USA. 